\title{
Special issue on recent advances in mobile service computing and applications
}

\author{
Honghao Gao' ${ }^{1}$ Yuyu Yin $^{2} \cdot$ Yucong Duan ${ }^{3}$
}

Published online: 27 November 2019

(C) Springer Science+Business Media, LLC, part of Springer Nature 2019

With the development of 5G technology and edge computing, the mobile application has greatly facilitated in our daily life. As core problems, how to make mobile service computing smart and trust and how to provide high-quality services for mobile applications are increasing attention from both the industry and academia. This special issue aims to present the state-of-the-art research on mobile service computing, as well as to organize a forum for experts to disseminate their recent advances and views on future perspectives in the field of mobility, limited capability, restricted power, unguaranteed security. After strict review, this special issue features six selected papers with high quality.

The first paper titled Local community detection for multi-layer mobile network based on the trust relation proposes a local community detection algorithm for multilayer complicated network based on the trust relation (MTLCD) to constrain the node tensor. They compare the performance of the proposed algorithm with other classic network clustering algorithms, such as GL, LART. Their algorithm can be used to effectively identify the local community.

The second paper titled Distributed Machine Learning Load Balancing Strategy in Cloud Computing Services implements an A-DSP model based on AdaptFR load

Honghao Gao

gaohonghao@shu.edu.cn

Yuyu Yin

yinyuyu@hdu.edu.cn

Yucong Duan

duanyucong@hotmail.com

1 Computing Center, Shanghai University, Shanghai, China

2 College of Computer, Hangzhou Dianzi University, Hangzhou, China

3 College of Information Science and Technology, Hainan University, Haikou, China balancing strategy. The method can dynamically adjust the communication strategy between the computing node and the parameter server according to the performance of the cluster nodes. Thus, this model improves the training speed.

The third paper titled Computation Offloading for Multimedia Workflows with Deadline Constraints in Cloudlet-Based Mobile Cloud proposes a multimedia workflow offloading method in the cloudlet-based MCC environment. They first discuss how to model multimedia applications and analyze the model from two aspects of time and energy consumption. And then, they use the NSDE algorithm to optimize the model and minimize the energy consumption with the constraints of meeting the deadline of each multimedia workflow.

The fourth paper titled Privacy Preserving Semantic Trajectory Data Publishing for Mobile Location-based Services considers the POI information and the users' motion modes such as walking, running, driving. The semantic trajectory anonymizing based on the k-anonymity model is proposed. It can form sensitive areas containing k-1 POI points that are similar to the sensitive points. Then, trajectory ambiguity is executed based on the motion modes, road network topologies, and road weights in the sensitive area.

The fifth paper titled A Novel Approach of Dynamic Base Station Switching Strategy based on Markov Decision Process for Interference Alignment in VANETs uses a Markov Decision Process (MDP) model for multi-antenna vehicles to estimate whether it is appropriate to be a dynamic base station. Monte Carlo Tree Search (MCTS) algorithm is introduced to derive MDP policy. And then, the $\mathrm{V} 2 \mathrm{~V}$ Interference Alignment (V2V-IA) model is constructed for a dynamic base station to obtain the IA scheme to manage $\mathrm{V} 2 \mathrm{~V}$ communications and IA in VANETs.

The last paper titled Recent Advances in Consensus Protocols for Blockchain: A Survey utilizes a thorough classification to explain current consensus protocols in the 
Blockchain system, presents the characteristics of mainstream protocols, such as PoW, PoS, DPoS, PBFT, and analyzes the strengths and weaknesses of them. Then they evaluate the performance qualitatively and quantitatively.

Acknowledgement The guest editors are thankful to our reviewers for their effort in reviewing the manuscripts. We thank the Edit-in-Chief, Dr. Imrich Chlamtac, for his supportive guidance during the entire process. We also thank you for the support from the National Natural Science Foundation of China (NSFC) under Grant No. 61902236.

Publisher's Note Springer Nature remains neutral with regard to jurisdictional claims in published maps and institutional affiliations.

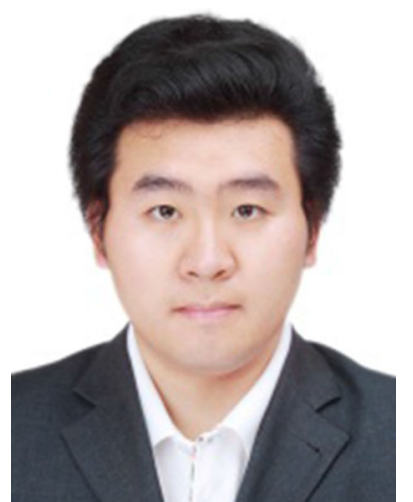

Prof. Dr. Honghao Gao received the Ph.D. degree in Computer Science and started his academic career at Shanghai University in 2012. He is an IET Fellow (FIET), BCS Fellow (FBCS), EAI Fellow (FEAI), IEEE Senior Member (SMIEEE), CCF Senior Member (SMCCF), and CAAI Senior Member (SMCAAI). Dr. Gao is currently a Distinguished Professor with the Key Laboratory of Complex Systems Modeling and Simulation, Ministry of Education, China, and is a Research Fellow with the Software Engineering Information Technology Institute of Central Michigan University (CMU), USA. He is an Adjunct Professor of Gachon University, South Korea. Dr. Gao's research interests include service computing, model checking-based software verification, wireless network and IoT, and intelligent medical image processing. His researches are supported by National Natural Science Foundation of China (NSFC), Natural Science Foundation of Shanghai (NSFS), CERNET Innovation Project, and Foundation of Science and Technology Commission of Shanghai Municipality. Until now, he has approximately 40 publications in professional journals and more than 20 publications in scientific conferences including IEEE TII, IEEE TCSS, IEEE IOT, IEEE TETCI, IEEE ACCESS, FGCS, MONET, COMCOM, IJDSN, JWCN, WCMC, IJSEKE, Autosoft, MIS, CI, FCS, SCN, AMIS, IJCM, JIT, ICSW, SCC, SEKE, Mobiquitous, TASE, CollaborateCom, and SNPD, obtained 13 patent applications and registered 8 software copyrights in China involving intelligent vehicle, searching engine, cloud rendering, service composition verification and testing, and IoT application under IPv4/IPv6. He has been served as Lead Guest Editor for ACM TOMM, IEEE TITS, IEEE ACCESS, Wiley ETT, ComSIS, CI, IJDSN, MONET, WINE, JIT, JAIHC, JOEUC, JMIHI, CAI, and organized more than 20 International Conferences and Workshops, such as CollaborateCom, ChinaCom, TRIDENTCOM, Broadnest, MobiCASE, ISC, IITC, FMSC, DISA, SSIA, IEEE/ACIS ICIS and ACTI.

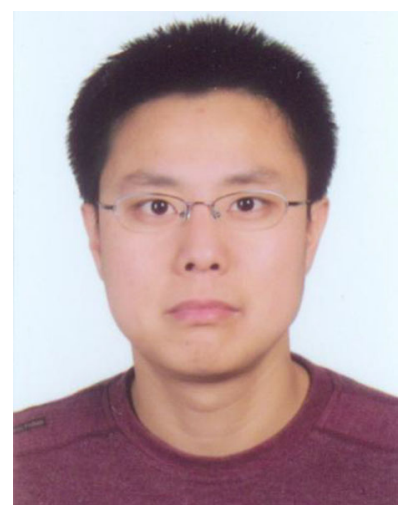

Prof. Dr. Yuyu Yin received the Ph.D. in Computer Science from Zhejiang University in 2010. He is currently an Associate Professor at the College of Computer in Hangzhou Dianzi University. His research interests include Service Computing, Cloud Computing, and Business Process Management. $\mathrm{He}$ is an EAI Fellow, IEEE Member, Senior Member of the China Computer Federation (CCF). During the past ten years, he has published more than 50 papers in journals and refereed conferences such as MONET, IEEE ACCESS, Sensors, Entropy, IJSEKE, Mobile Information Systems, ICWS, SEKE. He organized and chaired more than 10 international conferences and workshops, such as Mobicase 2019, Collaboratecom 2018, Tridentcom 2018/2019, FMSC2011-2017, DISA2012 2017-2018. Also he worked as EIC of EAI Endorsed Transactions on Internet of Things, as guest editor for Journal of Information Science and Engineering and International Journal of Software Engineering and Knowledge Engineering, and as reviewers for IEEE transaction on Industry Informatics, Journal of Database Management, Future Generation Computer Systems, etc.

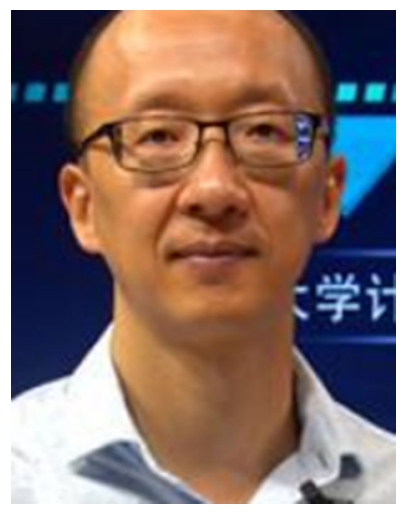

Prof. Dr. Yucong Duan is currently a Professor and vice director of Computer Science Department, Hainan University, P.R.China. He received a Ph.D. in software engineering from Institute of Software, Chinese Academy of Sciences, China in 2006. He was Post-doc fellow in School of Software, Tsinghua University, China from 2006 to 2007. He was a Post-doc fellow in the Software Engineering Laboratory at Pohang University of Science and Technology (POSTECH), South Korea, from 2007 to 2008. He was a lecturer of Biomedical Engineering Institute, Capital University of Medical Sciences, Beijing, China, from 2008 to 2009. He was a Post-doc fellow in Le2i, CNRS, University of Bourgogne, France, from 2009 to 2010. He was a postdoc in DISCO, University of Milano Bicocca, Milano, Italy, from 2011 to 2012. He has been a Senior member of IEEE. He joined in the CCF at 2013 and served as a committee member of the Service Computing professional committee of CCF. $\mathrm{He}$ has published more than 100 international journal or conference papers. His research interests include: theoretical and empirical software engineering, model driven software development, knowledge engineering, ontology modeling, service science, data cleaning of very large database, service/data quality evaluation, cognitive linguistics, service computing, SOA, cloud computing, etc. 